\title{
tmap: topological analysis of population-scale microbiome data
}

\author{
Tianhua Liao ${ }^{1, \dagger}$, Yuchen Wei ${ }^{2, \dagger}$, Mingjing Luo ${ }^{1}$, Guoping Zhao ${ }^{1,2,3,{ }^{*}}$, Haokui Zhou ${ }^{1,2, *}$ \\ ${ }^{1}$ Institute of Synthetic Biology, Shenzhen Institutes of Advanced Technology, Chinese \\ Academy of Sciences, Shenzhen 518055, China \\ ${ }^{2}$ Department of Microbiology, the Chinese University of Hong Kong, Hong Kong 999077, \\ China \\ ${ }^{3}$ Bio-Med Big Data Center, CAS Key Laboratory of Computational Biology, CAS-MPG \\ Partner Institute for Computational Biology, Chinese Academy of Sciences, Shanghai 200031, \\ China \\ * To whom correspondence should be addressed. \\ The authors wish it to be known that, in their opinion, the first two authors should be \\ regarded as Joint First Authors.
}

\begin{abstract}
Population-scale microbiome study poses specific challenges in data analysis, from enterotype analysis, identification of driver species, to microbiome-wide association of host covariates. Application of advanced data mining techniques to high-dimensional complex dataset is expected to meet the rapid advancement in large scale and integrative microbiome research. Here, we present tmap, a topological data analysis framework for population-scale microbiome study. This framework can capture complex shape of large scale microbiome data into a compressive network representation. We also develop network-based statistical analysis for driver species identification and microbiome-wide association analysis. tmap can be used for exploring variations in a population-scale microbiome landscape to study hostmicrobiome association.
\end{abstract}

Availability and implementation:

tmap is available at GitHub (https://github.com/GPZ-Bioinfo/tmap), accompanied with online documentation and tutorial (http://tmap.readthedocs.io).

Contact: hk.zhou@siat.ac.cn

\section{Introduction}

Population-scale microbiome studies have led to the identification of host factors associated with gut microbiome (Falony et al., 2016), characterization of gut microbiome enterotypes and their driver microbial species (Arumugam et al., 2011). The application of microbiomewide association analysis (MWAS) to population cohorts has revealed marker species associated with diseases (Gilbert et al., 2016). Standard microbiome data analysis relies on ordination techniques and regression analysis to discover variations of microbiome community and identify their association with host phenotypes (Knight et al., 2018). With the trends in conducting population-scale studies, multi-omics data integration and meta-analysis, rapidly advances in microbiome analysis methods and standards are expected, and will benefit from machine learning techniques in analyzing large scale high dimensional dataset (Mallick et al., 2017). 
Topological data analysis (TDA) provides a promising technique for analyzing large scale complex data. The most popular Mapper algorithm is effective in distilling data-shape from high dimensional space, and provides a compressive network representation (Singh et al., 2007; Lum et al., 2013). This algorithm has demonstrated its superior to traditional dimension reduction methods in analyzing biological and medical data, in which many of the challenges are similar to microbiome studies, such as subtyping of disease (Li et al., 2015), clustering of cancer groups, and identification of associated gene features (Nicolau et al., 2011).

Here, we present the tmap software as an implementation of the TDA Mapper framework for population-scale microbiome data analysis. We developed tmap to enable easy adoption of TDA in microbiome data analysis pipeline, providing network-based statistical methods for enterotype analysis, driver species identification, and microbiome-wide association of host meta-data. As demonstrated in this study, by re-analyzing the FGFP microbiome data (Falony et al., 2016), tmap is a promising microbiome data analysis framework for both large scale exploratory analysis and network-based hypothesis testing in population-scale microbiome study.

\section{Workflow and implementation}

\section{1 tmap workflow}

We implemented tmap as a Python package, consisting of modules and classes for major steps of the Mapper algorithm (Fig.1). Design of the modules and classes was motivated to provide flexible and consistent application programming interfaces (APIs) for the workflow (online documentation). By using the APIs, the workflow can be extended to incorporate machine learning methods from other Python packages, such as Scikit-learn (Pedregosa et al., 2011). Furthermore, tmap APIs allow for easy integration of the workflow into other microbiome analysis pipelines, such as QIIME (Caporaso et al., 2010). tmap has several advantages in microbiome data analysis, from the use of precomputed beta-diversity distance matrix, helper functions for TDA parameter selection, to the downstream network-based species enrichment and meta-data association analysis (online documentation). tmap provides an integrated and streamlined workflow to take the results from standard microbiome data analysis pipelines (such as QIIME) to advanced analysis and generates insights from microbiome data.

\subsection{Steps of tmap analysis}

A typical tmap analysis consists of four major steps, as illustrated in Fig.1. The first step is to project microbiome data into a low-dimensional space using dimension reduction methods with a specified distance metric. The second step is to make topological covering and clustering of the projected data. Depending on sample size and required resolution of microbiome variations, parameters of covering and clustering should be chosen and examined carefully. We provide a detailed explanation and guideline in the online documentation for these parameters. The third step is to generate, visualize and explore the microbiome TDA network. This step allows for discovering of patterns in microbiome variations and for visualizing how the pattern changes in the network along with meta-data, by mapping colors on the network. At last, network analysis is performed to identify driver species responsible for the observed community variation, and for metadata association analysis. 


\subsection{Network enrichment analysis of driver species}

Enterotypes and driver species can be identified from microbiome data using ordination and regression analysis. In tmap, we used a network enrichment technique for driver species analysis, based on a network representation of microbiome variations, which is adapted from the spatial analysis of functional enrichment (SAFE) algorithm (Baryshnikova, 2016) (Supplementary Material). Our approach successfully recovered all the top ten driver species from the FGFP cohort. Our approach also identified new driver species that are separated into multiple node clusters, which are also enriched in the network (Supplementary Table 2 and Fig. S1). The multi-cluster pattern of these driver species could be hard to detect with linear regression methods and therefore missed from the FGFP study. Another advantage of network enrichment analysis is a direct assignment and visualization of enterotype for each node (Supplementary Fig. S2).

\subsection{Network-based microbiome-wide association analysis}

tmap provides a novel alternative to standard MWAS by using network-based association analysis. Instead of association with individual samples, tmap uses SAFE enrichment scores at node level, which is a group of samples of highly similar microbiome composition. Application of this approach to the FGFP cohort successfully identified most of the reported associations, with improved power and effect sizes (Supplementary Table 4). We have also identified new associations not reported in the original study (Supplementary Fig. S4).

\section{Conclusion}

tmap is an integrated and advanced framework for topological analysis of microbiome data for population-scale study. The framework captures the topological shape of microbiome composition and variation into a network representaion, followed by network-based statistical analysis. We expect that tmap will enable researchers to obtain more insights from microbiome data, from enterotype analysis, driver species identification, understanding of microbiome landscape, to microbiome-wide association analysis.

\section{Acknowledgements}

We thank Huansheng Gao and Peijian Qiu for their help in improving the Python codes for network plotting and coloring.

\section{Funding}

This work was supported by Shenzhen Science and Technology Innovation Committee (Basic Science Research Grant JCYJ20170818154941048 to Zhou Haokui), National Key R\&D Program of China (2017YFC0907505), Key Research Program of the Chinese Academy of Sciences (KFZD-SW-219-5) and International Partnership Program of Chinese Academy of Sciences (153D31KYSB20170121). This work was also funded by Shenzhen Peacock Team Plan (KQTD2015033117210153 and KQTD2016112915000294), Engineering Laboratory for Automated Manufacturing of Therapeutic Synthetic Microbes (Shenzhen development and reform commission No. [2016]1194) and National Nature Science Foundation of China (No.31570095 and 31500104). 


\section{References}

Arumugam,M. et al. (2011) Enterotypes of the human gut microbiome. Nature, 473, 174-180. Baryshnikova,A. (2016) Systematic Functional Annotation and Visualization of Biological Networks. Cell Systems, 2, 412-421.

Caporaso,J.G. et al. (2010) QIIME allows analysis of high-throughput community sequencing data. Nat. Methods, 7, 335-336.

Falony,G. et al. (2016) Population-level analysis of gut microbiome variation. Science, 352, $560-564$.

Gilbert,J.A. et al. (2016) Microbiome-wide association studies link dynamic microbial consortia to disease. Nature, 535, 94-103.

Knight,R. et al. (2018) Best practices for analysing microbiomes. Nature Reviews Microbiology, 16, 410-422.

Li,L. et al. (2015) Identification of type 2 diabetes subgroups through topological analysis of patient similarity. Sci Transl Med, 7, 311ra174-311ra174.

Lum,P.Y. et al. (2013) Extracting insights from the shape of complex data using topology. Sci Rep, 3, 1236.

Mallick,H. et al. (2017) Experimental design and quantitative analysis of microbial community multiomics. Genome Biol., 18, 260.

Nicolau,M. et al. (2011) Topology based data analysis identifies a subgroup of breast cancers with a unique mutational profile and excellent survival. Proc. Natl. Acad. Sci. U.S.A., 108, 7265-7270.

Pedregosa,F. et al. (2011) Scikit-learn: Machine Learning in Python. J. Mach. Learn. Res., 12, 2825-2830.

Singh,G. et al. (2007) Topological Methods for the Analysis of High Dimensional Data Sets and 3D Object Recognition. pp. 91-100.

\section{Figure legend}

Fig.1. tmap workflow and steps of topological analysis of microbiome data. Starting with inputs of microbiome data (OTU table, beta-diversity distance matrix and sample metadata), tmap proceeds with data projection, covering, clustering and TDA network construction. Identification of driver species and association analysis of metadata are based on the TDA network structure, by calculating the SAFE network enrichment score. 


\section{Covering and Clustering}
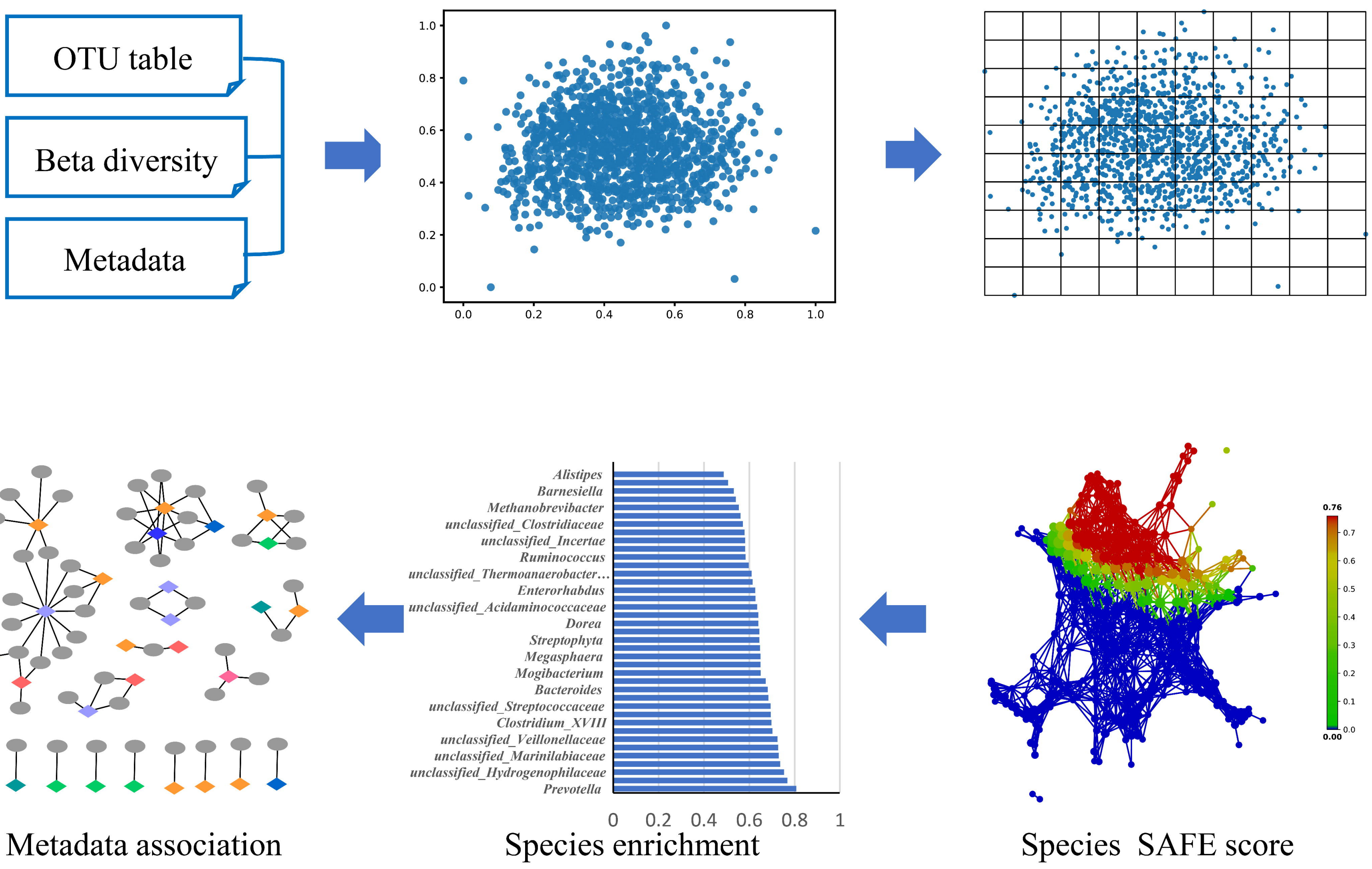

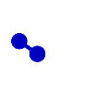

Species SAFE score

TDA network

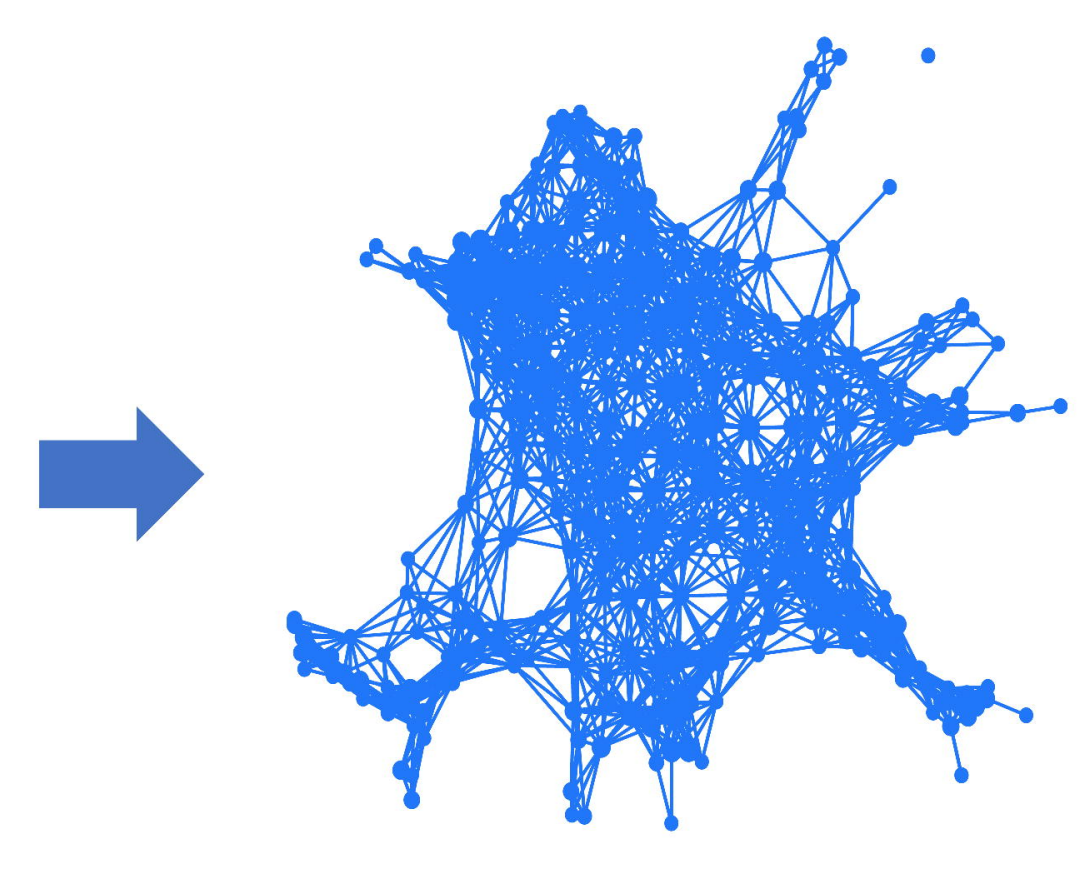

$a$

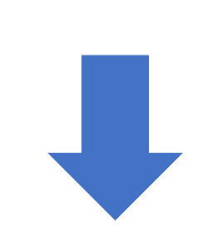

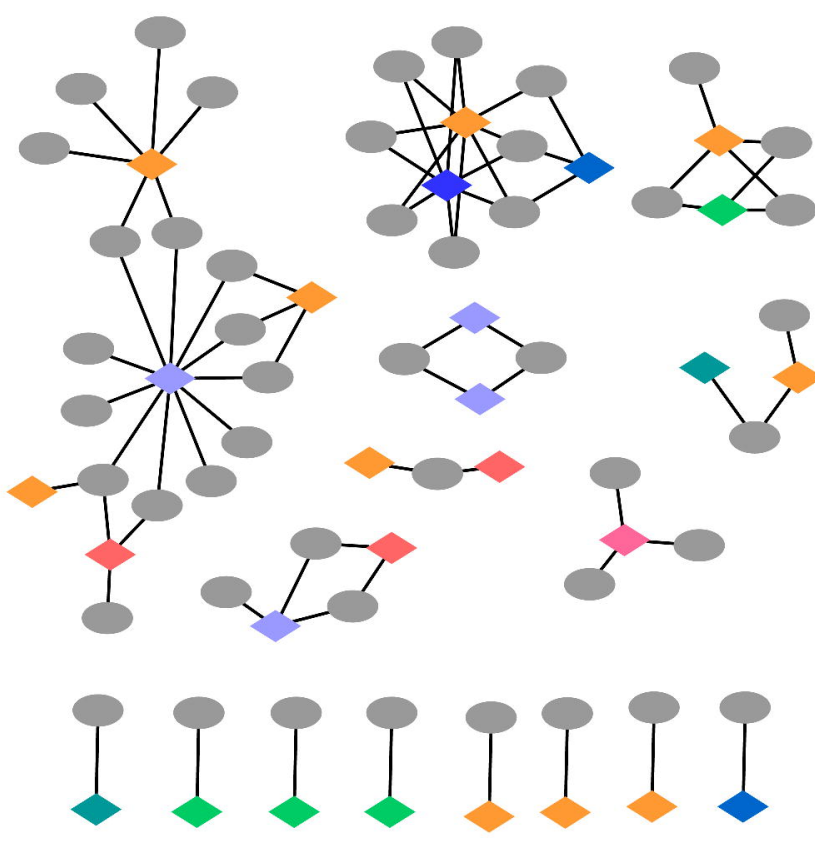

Metadata association

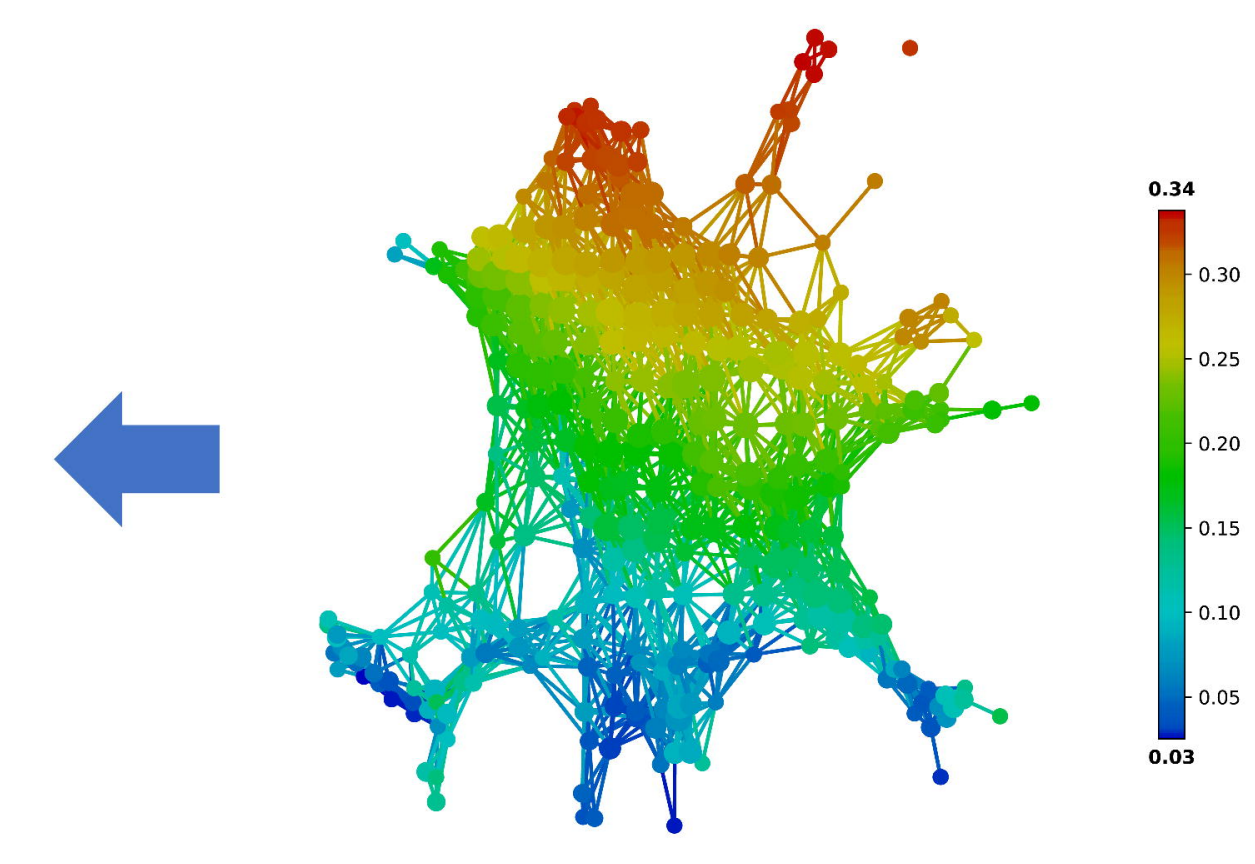

Species abundance 